Vietnam Journal of Mechanics, VAST, Vol. 42, No. 3 (2020), pp. 337-339

DOI: https://doi.org/10.15625/0866-7136/15494

Dedicated to Professor J.N. Reddy on the Occasion of His $75^{\text {th }}$ Birthday

\title{
NOTE ON EXTENSION OF THE YOUNG'S FORMULA TO INHOMOGENEOUS COLUMNS
}

\author{
Isaac Elishakoff ${ }^{1, *}$ \\ ${ }^{1}$ Department of Ocean and Mechanical Engineering, Florida Atlantic University, Boca Raton, FL 33431-0991, USA \\ *E-mail: elishako@fau.edu
}

Received: 08 September 2020 / Published online: 27 September 2020

\begin{abstract}
In this Note the generalization of the celebrated formula by Young, applicable to homogeneous columns, is provided for inhomogeneous columns.

Keywords: initial imperfection, Young's formula, inhomogeneous columns.
\end{abstract}

\section{INTRODUCTION}

In 1897, Thomas Young (1773-1829) derived the following formula for the total deflection of the initially bent uniform homogeneous column [1]

$$
w=\frac{w_{0}}{1-P / P_{c r, 1}},
$$

where $P$ is the axial loading applied to the column, $P_{c r, 1}$ is its critical fundamental buckling value $\pi^{2} D / L$, pertaining to the pinned-pinned column, $w_{0}$ is the initial deflection, $w=$ total deflection, $D=E I=$ flexural rigidity, $L=$ length. Bažant and Cedolin [2] state that Thomas Young [1] "was the first to take into account the presence of imperfection". In Eq. (1) the initial deflection has a sinusoidal shape

$$
w_{0}(x)=A \sin \pi \xi,
$$

where $A$ is its amplitude, $\xi=x / L$ is the non-dimensional axial coordinate. In this note we establish that analogous formula folds for inhomogeneous columns, namely those with variable flexural rigidity, $D(x)$.

\section{BASIC EQUATIONS}

Consider the initially bent column, pinned at its both ends. We denote the initial deflection by $w_{0}(\xi)$ and the additional deflection by $w(\xi)$. The governing differential equation reads

$$
D(\xi) w^{\prime \prime}+P L^{2}\left(w_{0}+w\right)=0,
$$

where primes denote differentiation with respect to $\xi$.

We are interested, in particular, in polynomial representation of the initial and additional deflections $w_{0}$ and $w$

$$
\begin{aligned}
& w_{0}(\xi)=A\left(\xi^{4}-2 \xi^{3}+\xi\right), \\
& w(\xi)=B\left(\xi^{4}-2 \xi^{3}+\xi\right),
\end{aligned}
$$

where $A$ is a given coefficient resulting from measured initial imperfections. Eq. (4) satisfies the boundary conditions at both ends of the column

$$
\begin{aligned}
& w(\xi)=0, \text { at } \xi=0 \text { and } \xi=1 \\
& w^{\prime \prime}(\xi)=0, \text { at } \xi=0 \text { and } \xi=1
\end{aligned}
$$

(C) 2020 Vietnam Academy of Science and Technology 
We solve the problem using the semi-inverse method; the problem is posed as follows: Find the flexural rigidity $D(\xi)$ such that the initial imperfections given in Eq. (4) result in additional displacements specified in Eq. (5). This method closely follows some recent vibration, and buckling problems solved in the monograph [3].

Polynomial expression for the flexural rigidity that is compatible with postulated functions in Eq. (4) and (5) reads

$$
D(\xi)=b_{0}+b_{1} \xi+b_{2} \xi^{2}
$$

where $b_{0}, b_{1}$ and $b_{2}$ are sought coefficients. Substitution of Eqs. (4), (5) and (8) into Eq. (3) leads to the following algebraic equation

$$
12 B\left[-b_{0} \xi+\left(b_{0}-b_{1}\right) \xi^{2}+\left(b_{1}-b_{2}\right) \xi^{3}+b_{2} \xi^{4}\right]+P L^{2} A\left(\xi^{4}-2 \xi^{3}+\xi\right)=-P L^{2} B\left(\xi^{4}-2 \xi^{3}+\xi\right) \text {. }
$$

This equation holds if the coefficients in front of $\xi^{j},(j=0,1,2,3)$ vanish. The requirement that the coefficient in front of $\xi^{4}$ is zero, leads to the equation

$$
12 B b_{2}+P L^{2} A=-P L^{2} B,
$$

leading to

$$
B=-\frac{P L^{2}}{12 b_{2}+P L^{2}} A
$$

We note that the buckling load of the ideal structure equals [3]

$$
P_{c r}=-12 b_{2} / L^{2} \text {. }
$$

Hence Eq. (11) can be rewritten as follows

$$
B=\frac{\alpha A}{1-\alpha},
$$

where $\alpha$ is the ratio

$$
\alpha=P / P_{c r, 1} .
$$

Demanding the coefficient in front of $\xi^{3}$ to vanish leads to

$$
12 B\left(b_{1}-b_{2}\right)=2 P L^{2}(A+B) \text {. }
$$

Taking into account Eqs. (11)-(14) results in

$$
b_{1}=-b_{2} \text {. }
$$

Since there is only one term $\left(b_{0}-b_{1}\right) \xi^{2}$ containing $\xi^{2}$, we conclude that $b_{0}=b_{1}=-b_{2}$. Hence, the flexural rigidity becomes

$$
D(\xi)=-\left(1+\xi-\xi^{2}\right) b_{2}
$$

In order the physical realizability condition $D(\xi)>0$ to be fulfilled, $b_{2}$ must be a negative quantity. Then, Eq. (17) can be rewritten as

$$
D(\xi)=\left(1+\xi-\xi^{2}\right)\left|b_{2}\right|
$$

The additional deflection function becomes, in view of Eqs. (5) and (13)

$$
w(\xi)=\frac{\alpha A}{1-\alpha}\left(\xi^{4}-2 \xi^{3}+\xi\right) .
$$

The total deflection reads

$$
w_{T}(\xi)=w_{0}(\xi)+w(\xi)=\left(A+\frac{\alpha A}{1-\alpha}\right)\left(\xi^{4}-2 \xi^{3}+\xi\right)=\frac{A}{1-\alpha}\left(\xi^{4}-2 \xi^{3}+\xi\right) .
$$

In view of Eq. (4) we conclude that

$$
w_{T}(\xi)=\frac{w_{0}(\xi)}{1-P / P_{c r, 1}},
$$

which coincides with Eq. (1) by Young. Thus, Young's formula turns out to be fully applicable to an inhomogeneous column with a specialized initial imperfection. Note that as the magnitude of the force 
$P$ tends to the value $P_{c r, 1}$ the magnitude of $w_{T}(x)$ becomes arbitrarily large. This conclusion parallels the conclusion valid for the uniform homogeneous column.

\section{CONCLUSION}

In recent careful review of the subject of elastic stability Bažant [4] writes, referring to Young's formula for the uniform homogeneous column: "While this formula is exact only for initial sinusoidal curvature, if represents an asymptotic approximation near $P_{c r, 1}$ for all kinds of initial imperfections. Therefore, it has recently been adopted as universal basis of design code ..."

The present study shows an remarkable fact: Whereas Young's formula is valid for initial sinusoidal curvature for the uniform homogeneous column, it equally holds for inhomogeneous column with initial polynomial curvature.

\section{ACKNOWLEDGEMENTS}

Author wishes Prof. J. N. Reddy many more decades of fruitful work for the benefit of world's mechanics. Thanks are to reviewers for constructive comments.

\section{REFERENCES}

[1] T. Young. A course of lectures on natural philosophy and the mechanical arts. 2 Volumes, London, (1807).

[2] Z. P. Bažant and L. Cedolin. Stability of structures: elastic, inelastic, fracture, and damage theories, p. 22. Oxford University Press, New York, (1991).

[3] I. Elishakoff. Eigenvalues of inhomogeneous structures: unusual closed-form solutions. CRC Press, Boca Raton, (2004).

[4] Z. P. Bažant. Stability of elastic, anelastic, and disintegrating structures: a conspectus of main results. ZAMM-Journal of Applied Mathematics and Mechanics/Zeitschrift für Angewoandte Mathematik und Mechanik: Applied Mathematics and Mechanics, 80, (11-12), (2000), pp. 709-732. https://doi.org/10.1002/15214001(200011)80:11/12<709::aid-zamm709>3.0.co;2-9. 